\title{
miR-373 suppresses gastric cancer metastasis by downregulating vimentin
}

\author{
YINGHONG SHI $^{1 *}$, HUI SHI $^{*}$, BIN ZHANG ${ }^{1 *}$, YONGMIN YAN $^{1}$, XINYE HAN $^{1}$, \\ WENQIAN JIANG $^{1}$, HUI QIAN ${ }^{1}$ and WENRONG XU ${ }^{1,2}$ \\ ${ }^{1}$ Key Laboratory of Laboratory Medicine of Jiangsu Province, School of Medicine, Jiangsu University, Zhenjiang, \\ Jiangsu 212013; ${ }^{2}$ The Affiliated Hospital of Jiangsu University, Zhenjiang, Jiangsu 212000, P.R. China
}

Received July 12, 2017; Accepted November 17, 2017

DOI: $10.3892 / \mathrm{mmr} .2017 .8291$

\begin{abstract}
MicroRNA-373 (miR-373) has been reported to be an oncogene in a number of solid human tumors. However, the role of miR-373 in gastric cancer has not been completely elucidated and the mechanisms remain unclear. In the present study, we compared miR-373 expression between clinical gastric cancer tissues and paired non-tumorous tissues by reverse transcription-quantitative polymerase chain reaction. The impact of miR-373 on proliferation, migration and invasion in gastric cancer cells was additionally investigated. Hsa-miR-373 mimics were applied to mimic the function of endogenous miR-373. A colony formation assay and flow cytometry were performed to analyze the proliferation of gastric cancer cells. Wound healing and Transwell invasion assays were employed to detect the migratory and invasive abilities of gastric cancer cells. Western blotting was used to test the expression of epithelial-mesenchymal transition-associated proteins. The results demonstrated that the level of miR-373 in gastric cancer was upregulated compared with paired non-tumorous tissues. It was confirmed that miR-373 inhibited the migration and invasion of the gastric cancer cell lines SGC-7901 and HGC-27 by downregulating vimentin expression. The results of the present study demonstrated an oncogenic role of miR-373 in the metastasis of human gastric cancer, and may provide a novel therapeutic strategy for gastric cancer.
\end{abstract}

Correspondence to: Professor Wenrong $\mathrm{Xu}$, Key Laboratory of Laboratory Medicine of Jiangsu Province, School of Medicine, Jiangsu University, 301 Xuefu Road, Zhenjiang, Jiangsu 212013, P.R. China

E-mail:icls@ujs.edu.cn

${ }^{*}$ Contributed equally

Key words: gastric cancer, microRNA-373, metastasis, vimentin, proliferation

\section{Introduction}

Gastric cancer is the fourth most common cancer and the second most common cause of cancer-associated mortality in the world (1-3). Gastric cancer remains difficult to cure, primarily since the majority patients present with advanced disease or even suffer from gastric cancer metastasis. Therefore, the mechanisms of gastric cancer invasion and migration remain to be elucidated.

MicroRNAs (miRNAs/miRs) are small non-coding RNAs, which are approximately 20 nucleotides (nt) long and are involved in vast range of cellular processes (4-6). miRNAs negatively regulate gene expression by repressing translation or inducing target mRNA degradation $(5,6)$, processes which are notably involved in cell proliferation, differentiation and apoptosis (7). miRNAs have been observed to serve roles in numerous types of cancer. Increasing evidence has indicated that miRNAs may function as oncogenes or tumor suppressor genes, which are crucial in the initiation and progression of human malignancies (8). miR-21 is an extensively-researched oncogenic miRNA in a number of solid human tumors, including non-muscle-invasive bladder cancer (9), colorectal cancer (10), non-small cell lung cancer (11) and gastric cancer (12). miR-21 promoted cell growth and invasion by repressing phosphatidylinositol 3,4,5-trisphosphate 3-phosphatase and dual-specificity protein phosphatase PTEN in colorectal cancer (13), similar to the impact of miR-21 on gastric cancer (14). Recently, miR-373 was demonstrated to be an important gene in numerous human malignant tumors (15-17), which provoked widespread attention.

Hsa-miR-373 belongs to the miR-371-3 gene cluster, which is transcribed into the primary transcript pri-miR-3771-373. The pri-miR-371-3 is processed into three pre-miRNAs (pre-miR371, pre-miR372 and pre-miR-373), giving rise to four mature miRNAs (miR-371, miR-372, miR-373 and miR-373*) (18). Hsa-miR-373, which was first reported to be a human embryonic stem cell (ESC)-specific miRNA (18). Subsequently, miR-373, consistent with miR-372, was identified to be an oncogene in the tumorigenesis of human testicular germ cell tumors, through the direct inhibition of large tumor suppressor $1 / 2$ expression (18). In 2011, Zhang et al (19) reported that the downregulation of hsa-miR-373 had the 
potential to predict recurrence risk in patients with gastric cancer following surgical resection. The following year, it was discovered that PR domain zinc finger protein 4, a recurrence risk biomarker in patients with gastric cancer, was regulated by miR-373 (20). An additional study revealed that miR-373 is upregulated in gastric adenocarcinoma tissue and gastric carcinoma cell lines, and it induces cell proliferation in gastric adenocarcinoma cells in vitro via the downregulation of broad-complex, tramtrack and Bric a brac/Poxvirus and zinc finger domain-containing adapter for cullin3-mediated RhoA degradation protein 2 (21). Although oncogenic roles of miR-373 have been demonstrated, the molecular mechanisms of miR-373 in gastric cancer metastasis remain unclear. Mmu-miR-294 is one member of the miR-290 family, which has been demonstrated to be the homologous gene family of the hsa-miR-371-3 gene family (22). Therefore, the role of mmu-miR-294 in mouse gastric cancer remains to be elucidated.

In the present study, the effect of hsa-miR-373/ mmu-miR-294 on gastric cancer cellular metastasis was investigated. The results demonstrated that hsa-miR-373 and mmu-miR-294 were able to suppress the metastasis of gastric cancer cells by negatively regulating the expression of vimentin. Furthermore, it was observed that hsa-miR-373 and mmu-miR-294 were able to promote gastric cancer cell proliferation. These results may provide a novel therapeutic strategy for gastric cancer with metastasis.

\section{Materials and methods}

Clinical gastric cancer tissues. The primary malignant gastric cancer tissues and their matching and adjacent non-tumorous tissues (located $>5-10 \mathrm{~cm}$ away from the primary site) were collected from 28 patients with gastric cancer undergoing surgery at the Affiliated Hospital of Jiangsu University from March 2016 to May 2017. (Zhenjiang, China). Informed consent was given by all patients examined. All samples were confirmed by pathological examination. Histological grade was defined according to the World Health Organization classification system. The tissues obtained were immediately stored at $-80^{\circ} \mathrm{C}$. The present study was approved by the Medical Ethics Committee of Jiangsu University.

Cell culture. Human gastric cancer cell lines SGC-7901 and $\mathrm{HGC}-27$, and mouse gastric cancer cell line MFC, were obtained from the Institute of Biochemistry and Cell Biology at the Chinese Academy of Sciences (Shanghai, China). These cell lines were cultured in RPMI-1640 medium (Gibco; Thermo Fisher Scientific, Inc., Waltham, MA, USA). All medium was supplemented with $10 \%$ fetal bovine serum (FBS; Gibco; Thermo Fisher Scientific, Inc.). Cells were incubated at $37^{\circ} \mathrm{C}$ in humidified air with $5 \% \mathrm{CO}_{2}$.

Reverse transcription-quantitative polymerase chain reaction (RT-qPCR) for $m R N A$ expression. Total RNA from human gastric tissues and gastric cancer cells was extracted using TRIzol reagent (Life Technologies; Thermo Fisher Scientific, Inc.). RT was performed using a HiScript 1st Strand cDNA Synthesis kit (Vazyme, Piscataway, NJ, USA) and the signal-stranded cDNA was obtained. RT was performed at $25^{\circ} \mathrm{C}$ for $5 \mathrm{~min}, 50^{\circ} \mathrm{C}$ for $15 \mathrm{~min}$ and $85^{\circ} \mathrm{C}$ for $5 \mathrm{~min}$. The qPCR reactions were performed using the QuantiTect SYBR Green PCR kit (Toyobo Co., Ltd., Osaka, Japan). Thermal cycling parameters were as follows: $94^{\circ} \mathrm{C}$ for $5 \mathrm{~min} ; 40$ cycles at $94^{\circ} \mathrm{C}$ for $30 \mathrm{sec}, 55^{\circ} \mathrm{C}$ for $30 \mathrm{sec}$ and $72^{\circ} \mathrm{C}$ for $30 \mathrm{sec}$; and $65-95^{\circ} \mathrm{C}$ for drawing the dissociation curve. The expression level of mRNA was normalized to the expression of $\beta$-actin (23). The primer sequences were as follows: Vimentin sense, 5'-ATACTGCTG GCGCACATCAC-3', and vimentin antisense, 5'-CCCTTT CCCCAGTTTTTAATAGG-3'; $\beta$-actin sense, 5'-GACCTG TACGCCAACACAGT-3', and $\beta$-actin antisense, 5 '-CTCAGG AGGAGCAATGATCT-3'.

RT-qPCR for miRNA expression. RT was performed using the miScript II RT kit (Qiagen China Co., Ltd., Shanghai, China) and the signal-stranded cDNA was obtained. RT was performed at $42^{\circ} \mathrm{C}$ for $60 \mathrm{~min}$, followed by an inactivation reaction at $70^{\circ} \mathrm{C}$ for $15 \mathrm{~min}$. The CFX96 Real Time Instrument (Bio-Rad Laboratories, Inc., Hercules, CA, USA) and miScript SYBR Green PCR kit (Qiagen China Co., Ltd.) were used for quantitative RNA detection. Thermal cycling parameters were as follows: $95^{\circ} \mathrm{C}$ for $5 \mathrm{~min} ; 40$ cycles at $94^{\circ} \mathrm{C}$ for $15 \mathrm{sec}, 55^{\circ} \mathrm{C}$ for $15 \mathrm{sec}$ and $72^{\circ} \mathrm{C}$ for $20 \mathrm{sec}$; and $65-95^{\circ} \mathrm{C}$ for drawing the dissociation curve. The relative expression levels of miRNA were normalized to the expression of small nuclear RNA U6 (23). PCR primers were as follows: miR-373 sense, 5'-CGC GCGAAGTGCTTCGATTT-3', and miR-373 antisense, 5'-GTGCAGGGTCCGAGGT-3'; U6 sense, 5'-GCTTCGGCA GCACATATACTAAAAT-3', and U6 antisense, 5'-CGCTTC ACGAATTTGCGTGTCAT-3'.

Transfection. miRNA mimics were purchased from Shanghai GenePharma Co., Ltd. (Shanghai, China), and Lipofectamine ${ }^{\circledR} 2000$ (Invitrogen; Thermo Fisher Scientific, Inc.) was used for transfection. The concentrations of hsa-miR-373 mimics or mmu-miR-294 mimics were 10, 12.5 and $25 \mathrm{nM}$. The same concentrations of mimic controls (NC) were used. The sequences of the oligonucleotides were as follows: miR-294 mimics NC sense, 5'-UUCUUCGAACGU GUCACGUTT-3', miR-294 mimics NC antisense, 5'-ACG UGACACGUUCGGAGAATT-3'; miR-373 mimics NC sense, 5'-UUCUCCGAACGUGUCACGUTT-3'; miR-373 mimics NC antisense, 5'-ACGUGACACGUUCGGAGAATT-3'; miR-373 mimics sense, 5'-GAAGUGCUUCGAUUUUGG GGUGU-3'; and miR-373 mimics antisense, 5'-ACCCCAAAA UCGAAGCACUUCUU-3'.

Cell cycle. A total of $1 \times 10^{5}$ SGC-7901, $1 \times 10^{5}$ HGC-27 or $1.5 \times 10^{5} \mathrm{MFC}$ cells were seeded in each well of a 6 -well plate 1 day prior to transfection, and transfected with miRNA mimics. A total of $6 \mathrm{~h}$ subsequently, cells were washed twice with PBS and placed in fresh medium containing $10 \%$ FBS for $48 \mathrm{~h}$. Cells were collected and washed twice with $\mathrm{PBS}$, and stained with $10 \mu \mathrm{g} / \mathrm{ml}$ propidium iodide (Sigma-Aldrich; Merck KGaA, Darmstadt, Germany) in $500 \mu \mathrm{l}$ PBS (containing $100 \mu \mathrm{g} / \mathrm{ml}$ RNase) for $30 \mathrm{~min}$ in the dark at room temperature. A filter screen was used to isolate suspended cells. The distribution of cells in the different phases of the cell cycle was detected using a flow cytometer 
Table I. Correlation between clinical pathological factors and miR-373 expression in patients with gastric cancer.

\begin{tabular}{|c|c|c|c|c|}
\hline \multirow[b]{2}{*}{ Factor } & \multirow[b]{2}{*}{$\begin{array}{c}\text { No. } \\
(n=28)\end{array}$} & \multicolumn{2}{|c|}{$\begin{array}{l}\text { miR-373 } \\
\text { expression }\end{array}$} & \multirow[b]{2}{*}{$\mathrm{P}$-value } \\
\hline & & $\begin{array}{l}\text { Low } \\
\text { group }\end{array}$ & $\begin{array}{l}\text { High } \\
\text { group }\end{array}$ & \\
\hline Age, years & & & & 0.6270 \\
\hline$\geq 60$ & 16 & 8 & 8 & \\
\hline$<60$ & 12 & 4 & 8 & \\
\hline Sex & & & & 0.4725 \\
\hline Male & 24 & 12 & 12 & \\
\hline Female & 4 & 0 & 4 & \\
\hline Size, cm & & & & 1.0000 \\
\hline$\geq 5$ & 18 & 8 & 10 & \\
\hline$<5$ & 10 & 4 & 6 & \\
\hline Histological grade & & & & 0.4589 \\
\hline Poorly + signet & 12 & 4 & 8 & \\
\hline Moderately + well & 16 & 8 & 8 & \\
\hline Stage & & & & 0.4725 \\
\hline $\mathrm{I} / \mathrm{II}$ & 4 & 0 & 4 & \\
\hline III/IV & 24 & 12 & 12 & \\
\hline $\mathrm{T}$ grade & & & & 0.4725 \\
\hline $\mathrm{T} 1+\mathrm{T} 2$ & 4 & 0 & 4 & \\
\hline $\mathrm{T} 3+\mathrm{T} 4$ & 24 & 12 & 12 & \\
\hline \multicolumn{5}{|c|}{ Lymph node metastasis } \\
\hline ( $\mathrm{N}$ factor) & & & & 0.0882 \\
\hline N0+N1 & 8 & 1 & 7 & \\
\hline $\mathrm{N} 2+\mathrm{N} 3$ & 20 & 11 & 9 & \\
\hline
\end{tabular}

Poorly, poorly differentiated gastric carcinoma of high malignant degree; Signet, signet-ring cell carcinoma of high malignant degree.

(FACS Calibur; BD Biosciences, Franklin Lakes, NJ, USA) and analyzed with a flow cytometry software (FlowJo v7.6.5; BD Biosciences).

Colony formation assay. A total of 1,000 SGC-7901 or HGC-27 cells were seeded in each well of a 6-well plate in triplicate and incubated at $37^{\circ} \mathrm{C}$ in a $5 \% \mathrm{CO}_{2}$ humidified incubator for 8 days. The medium was changed at 3-day intervals. At the end of the incubation period, the cultures were fixed with $4 \%$ paraformaldehyde for $30 \mathrm{~min}$ at room temperature and stained with crystal violet for $20 \mathrm{~min}$ at room temperature. Images were captured in five random fields (magnification, $\mathrm{x} 40$ ) using an inverted fluorescent microscope (Nikon Corporation, Tokyo, Japan).

Wound healing assay. SGC-7901, HGC-27 and MFC cells were seeded at a density of $2 \times 10^{5}, 3 \times 10^{5}$ and $6 \times 10^{5}$ cells/well, respectively, in 6-well plates and incubated at $37^{\circ} \mathrm{C}$ in $5 \%$ $\mathrm{CO}_{2}$ for $24 \mathrm{~h}$ to create confluent monolayers. The monolayers were scratched with a sterile pipette tip. To measure the mobility of cells, images were captured in five random fields

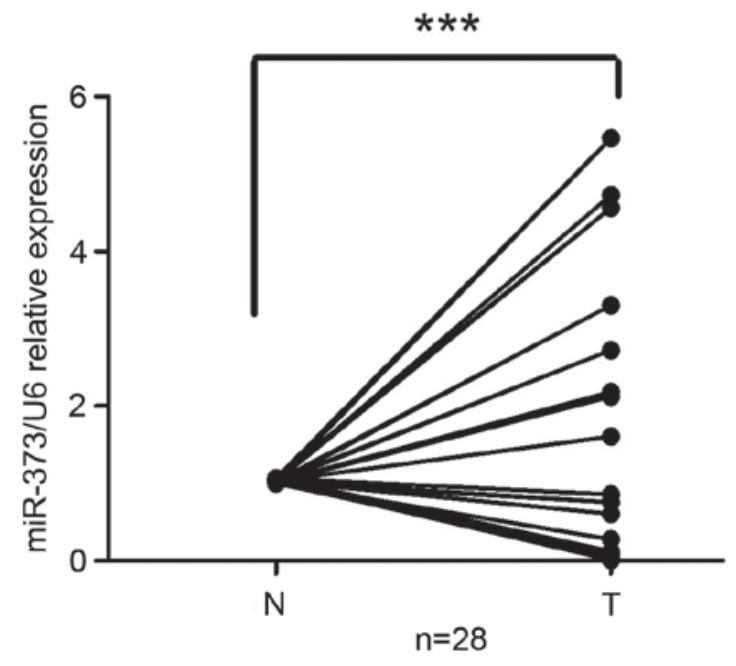

Figure 1. Relative level of miR-373 in gastric cancer tissues. Reverse transcription-quantitative polymerase chain reaction assay for miR-373 in tissues from 28 patients with gastric cancer. Data are presented as the fold change in miR levels relative to $\mathrm{N}$, which was set as $1 .{ }^{* * *} \mathrm{P}<0.001$. $\mathrm{N}$, adjacent normal tissue; $\mathrm{T}$, gastric cancer tissue; miR, microRNA.

(magnification, $\mathrm{x} 40$ ) at $12 \mathrm{~h}$ post-scratching using an inverted fluorescent microscope (Nikon Corporation).

Transwell invasion assay. The abilities of cell migration and invasion were detected by Transwell assay. All cells were seeded at $1 \times 10^{5}$ cells/well in each well of a 24 -well plate with FBS-free RPMI-1640 media in the upper chamber, and there was $500 \mu \mathrm{l}$ fresh medium containing $10 \%$ FBS in the lower chamber. Prior to staining with crystal violet for $20 \mathrm{~min}$ at room temperature, cells were incubated at $37^{\circ} \mathrm{C}$ in humidified air with $5 \% \mathrm{CO}_{2}$ for $12 \mathrm{~h}$. Images were captured in 5 random fields (magnification, x40) using an upright fluorescent microscope (Nikon Corporation).

In silico target prediction of miRNA. TargetScan 7.1 (www.targetscan.org) target prediction software was used to indicated the miRNAs that may potentially target the 3'untranslated region of vimentin.

Western blotting. The total protein in gastric cancer cells was extracted using a lysis buffer containing $50 \mathrm{mM} \mathrm{NaCl}$, $1 \mathrm{mM}$ ethylene glycol tetra-acetic acid, $0.1 \%$ SDS, $1 \mathrm{mM} \mathrm{NaF}$, $1 \mathrm{mM}$ Na3VO4, $1 \mathrm{mg} / \mathrm{ml}$ aprotinin and $1 \mathrm{mg} / \mathrm{ml}$ leupeptin in $10 \mathrm{mM}$ Tris (pH 7.4) and proteinase inhibitors (1 mM phenylmethylsulfonyl fluoride). Protein concentrations were determined using spectrophotometry (Nanodrop; Thermo Fisher Scientific, Inc.). A total of $30 \mathrm{ug}$ total protein were separated on $10 \%$ SDS-PAGE gels and transferred to nitrocellulose membranes (EMD Millipore, Billerica, MA, USA). Membranes were blocked with 5\% milk in PBS for $1 \mathrm{~h}$ at $37^{\circ} \mathrm{C}$ and incubated with monoclonal antibodies against $\beta$-actin (BS6007M), proliferating cell nuclear antigen (PCNA; BZ02108), vimentin (MB9006) and E-cadherin (LM0206; all obtained from Bioworld Technology, Inc., St Louis Park, MN, USA) at $4^{\circ} \mathrm{C}$ overnight, the dilutions of which were 1:2,000, 1:4,000, 1:600 and 1:500, respectively. Following incubation with the secondary antibodies [1:2,000; goat anti-mouse 
A

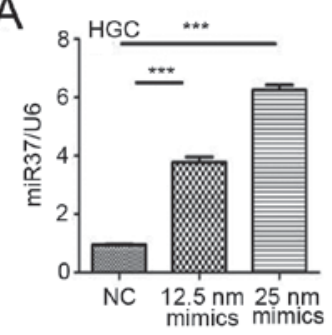

$\mathrm{Ba}$

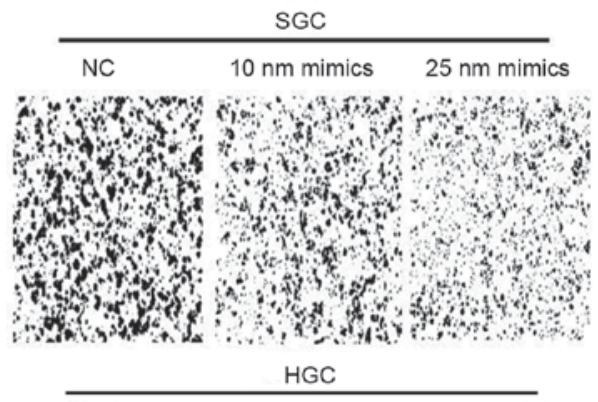

NC $\quad 12.5 \mathrm{~nm}$ mimics $25 \mathrm{~nm}$ mimics

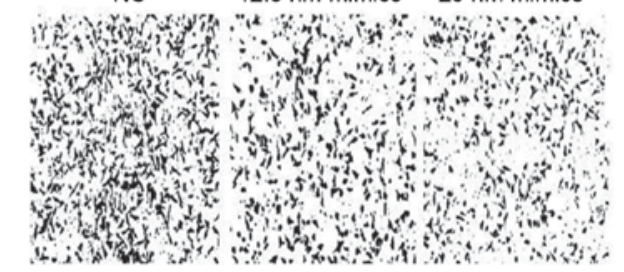

$\mathrm{Bb}$
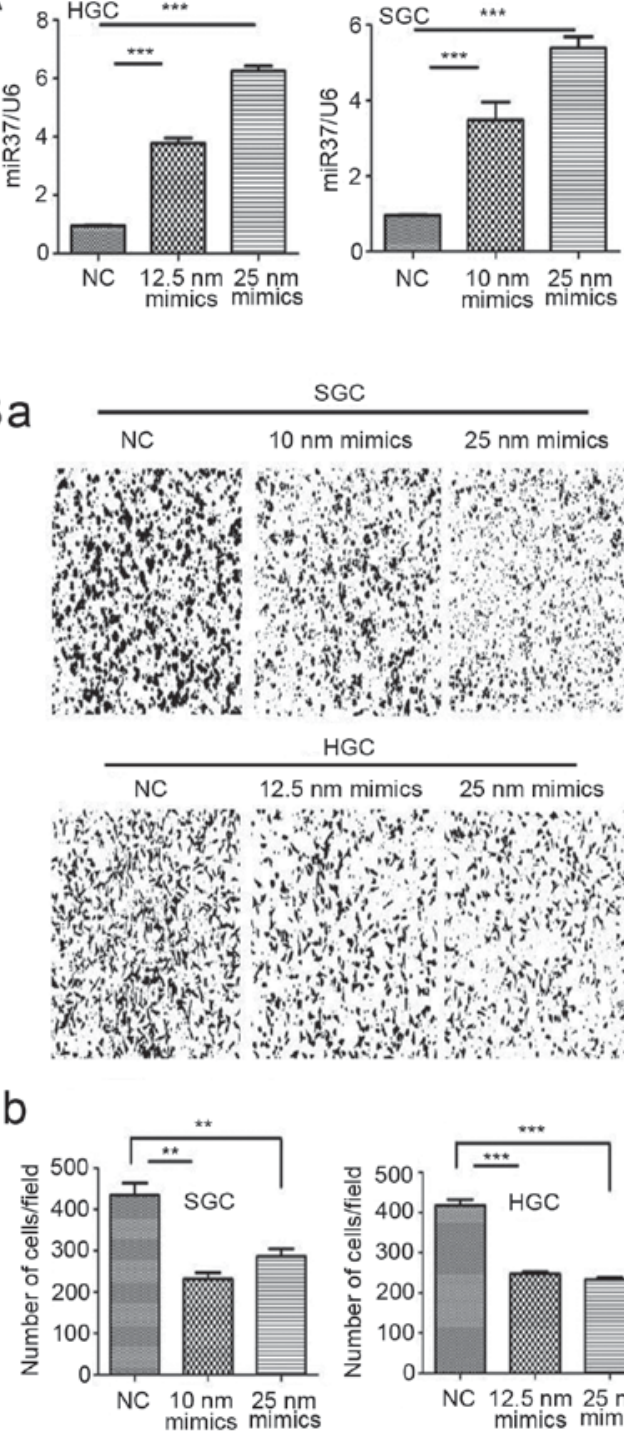

SGC

ixis

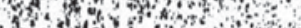

(1)

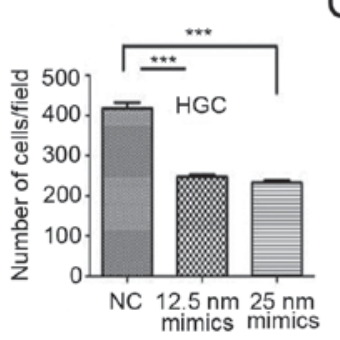

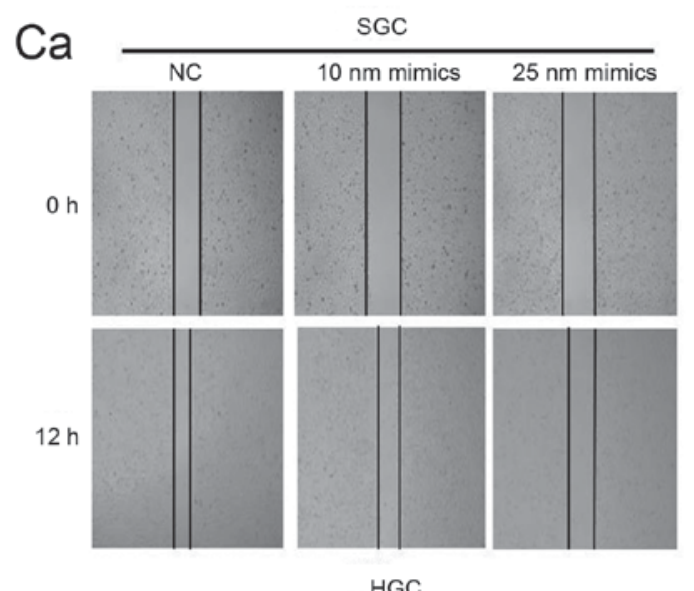
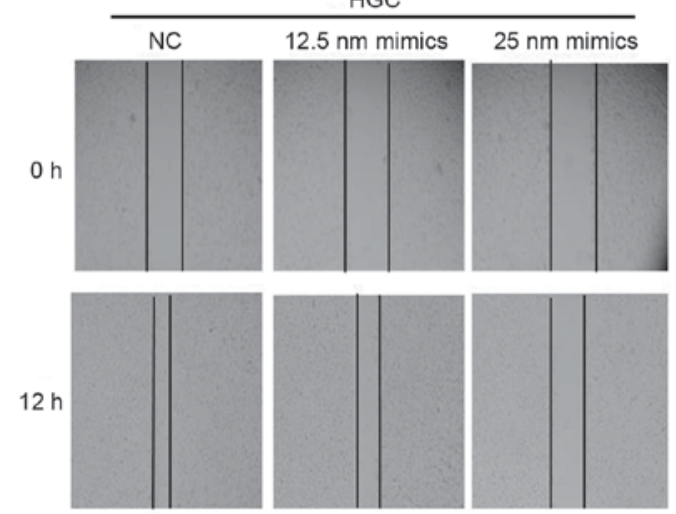

$\mathrm{Cb}$

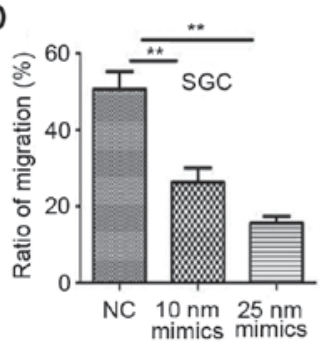

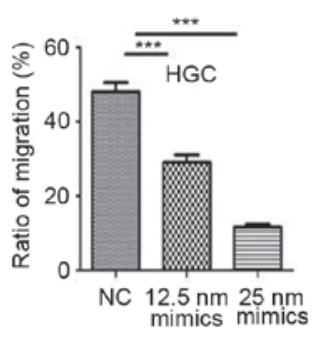

Figure 2. Hsa-miR-373 represses the migration and invasion of the gastric cancer cell lines SGC-7901 and HGC-27. (A) Reverse transcription-quantitative polymerase chain reaction analysis of miR-373 levels $48 \mathrm{~h}$ post-transfection with miRNA mimics. Data are presented as the fold change in miRNA levels relative to $\mathrm{NC}$, which was set as 1 . (Ba) Transwell invasion assay for cell invasion $48 \mathrm{~h}$ post-transfection (magnification, $\mathrm{x} 100$ ). (Bb)The number of migrated cells per field was quantified. (Ca) Wound healing assay for cell migration $48 \mathrm{~h}$ post-transfection (magnification, $\mathrm{x} 40$ ). (Cb) Ratios of migration were calculated. ${ }^{* *} \mathrm{P}<0.01,{ }^{* * *} \mathrm{P}<0.001$. NC, negative control; miRNA/miR, microRNA.

horseradish peroxidase immunoglobulin $\mathrm{G}(\mathrm{IgG}$; BS12478) and goat anti-rabbit horseradish peroxidase conjugated IgG (BS10043); all obtained from Bioworld Technology, Inc.] for $1 \mathrm{~h}$ at $37^{\circ} \mathrm{C}$, the signals were visualized using a chemiluminescent and fluorescent imaging system (EMD Millipore). $\beta$-actin was used as the loading control. The software used for analysis was ImageQuant LT 7.0 (GE Healthcare Bio-Sciences, Pittsburgh, PA, USA).

Statistical analysis. Statistical analysis of the differences in the expression levels of miR-373 in paired gastric cancer and adjacent non-tumorous tissues were analyzed using a paired t-test with Prism version 5. The correlation between miR-373 expression and clinicopathological factors was estimated using Fisher's exact test. Potential differences between groups with different treatments were analyzed using one-way analysis of variance, followed by a Dunnett t-test. All experiments were performed at least in triplicate $(n=3)$. Data are presented as the mean \pm standard deviation. All statistical analysis was performed with GraphPad Prism 5.0 software (GraphPad Software Inc., La Jolla, CA, USA). $\mathrm{P}<0.05$ was considered to indicate a statistically significant difference.

\section{Results}

Hsa-miR-373 is upregulated in human gastric cancer. To investigate the clinical significance of miR-373 in gastric cancer, miR-373 expression in 28 paired primary malignant gastric cancer tissues and adjacent non-tumorous tissues was compared. The results demonstrated that miR-373 was upregulated in gastric cancer tissues compared with normal gastric tissues $(\mathrm{n}=28, \mathrm{P}<0.001$; Fig. 1$)$. The correlation between miR-373 expression and clinical pathological 
$\mathrm{Aa}$
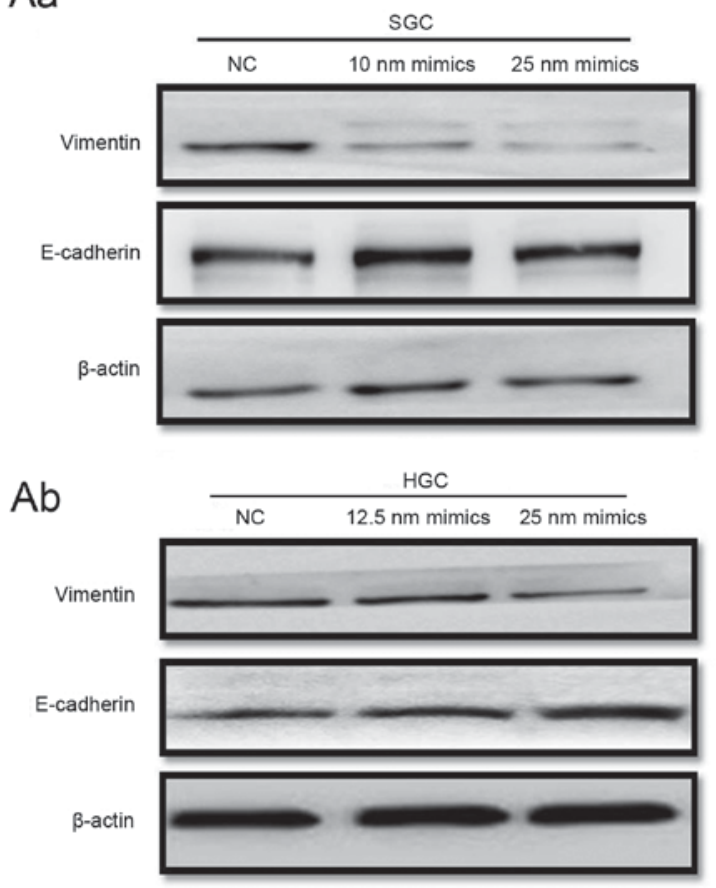

$\mathrm{Ba}$

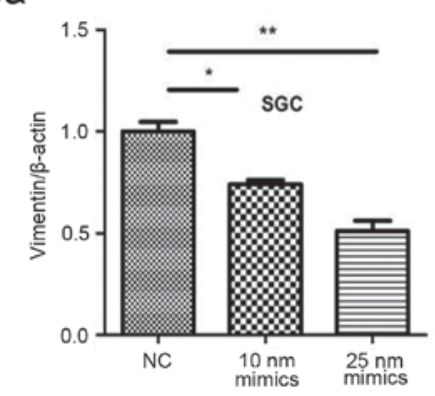

$\mathrm{Bb}$

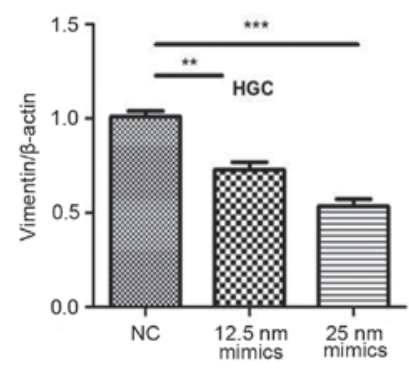

Figure 3. Hsa-miR-373 inhibits metastasis in SGC-7901 and HGC-27 cells via negative regulation of vimentin. Western blot analysis of vimentin, E-cadherin and $\beta$-actin proteins $48 \mathrm{~h}$ post-transfection with miRNA mimics, in (Aa) SGC-7901 and (Ab) HGC-27 cells. Reverse transcription-quantitative polymerase chain reaction analysis of vimentin levels $48 \mathrm{~h}$ post-transfection with miRNA mimics, in (Ba) SGC-7901 and (Bb) HGC-27 cells. Data are presented as the fold change in miRNA levels relative to $\mathrm{NC}$, which was set as $1 .{ }^{*} \mathrm{P}<0.05,{ }^{* *} \mathrm{P}<0.01,{ }^{* * *} \mathrm{P}<0.001$. NC, negative control; miRNA/miR, microRNA.

characteristics was analyzed. Gastric cancer tissues with a more than two-fold increase in miR-373 (relative to adjacent non-tumorous tissues) were defined as the high group, while those with a less than two-fold increase or a decrease in miR-373 (relative to adjacent non-tumorous tissues) were defined as the low group. The results demonstrated that relative miR-373 expression was increased in patients with N0/N1 stage lymph node metastasis (7/8), and a lower expression was observed in patients with N2/N3 stage lymph node metastasis (11/20). miRNA-373 levels were negatively correlated with lymph node metastasis (Table I). No correlation was observed between miR-373 expression and age, gender, tumor size, histological grade or clinical stage (Table I). Thus, it was hypothesized that miR-373 may be used to distinguish between cases of malignant primary gastric cancer with or without lymph node metastasis, and gastric cancer with a lower level of miR-373 may be more invasive.

Hsa-miR-373 represses the migration and invasion of gastric cancer SGC-7901 and HGC-27 cells. To investigate the role of miR-373 in gastric cancer cellular metastasis in vitro, SGC-7901 and HGC-27 cells were transfected with miR-373 mimics in vitro. The effect of miR-373 on invasion and migration were studied by Transwell invasion assay and wound healing assay, respectively. Following treatment with miR-373 mimics, the level of miR-373 in transfected cells increased (Fig. 2A). The results of the Transwell invasion assay demonstrated that SGC-7901 and HGC-27 invasion was decreased by miR-373 mimics compared with control cells (Fig. 2B). The results of the wound healing assay revealed that the wound size in miR-373-treated SGC-7901 and HGC-27 cells was larger compared with control cells (Fig. 2C). These findings suggested that miR-373 was able to inhibit the migration and invasion of SGC-7901 and HGC-27 cells.

Hsa-miR-373 downregulates vimentin expression in SGC-790 1 and HGC-27 cells. Previously miR-373 has been reported to be an oncogene, and to serve a role in tumor metastasis (24). However, the mechanism underlying the role of miR-373 in tumor metastasis remains unclear. Epithelial-mesenchymal transition has been demonstrated to serve an important role in promoting metastasis in gastric cancer (25). Vimentin is an important mesenchymal marker and E-cadherin is an epithelial marker. The effect of miR-373 on vimentin and E-cadherin expression was detected by western blotting. The results demonstrated that vimentin was downregulated in miR-373 mimics-treated cells compared with the negative control, and E-cadherin was increased (Fig. 3A). The mRNA expression of vimentin was additionally decreased in miR-373-treated cells compared with the negative control (Fig. 3B). These results revealed that miR-373 was able to downregulate vimentin expression in SGC-7901 and HGC-27 cells.

Mmu-miR-294 represses the metastasis of mouse gastric cancer MFC cells by inhibiting vimentin. Hsa-miR-373 belongs to the miR-371-3 gene cluster, which has been demonstrated to be the homologous gene family of the Mmu-miR-294 gene family (22). Mouse miR-294, a homologous gene of miR-373, 


\section{A}

Position 137-144 of VIM 3'UTR 5'...AAUAGAAAGAAGCUCAGCACUUA...

Mmu-miR-294-3p 3'UGUGUGUUUCCCU -- . UCGUAAA.

B

NC

$10 \mathrm{~nm}$ mimics
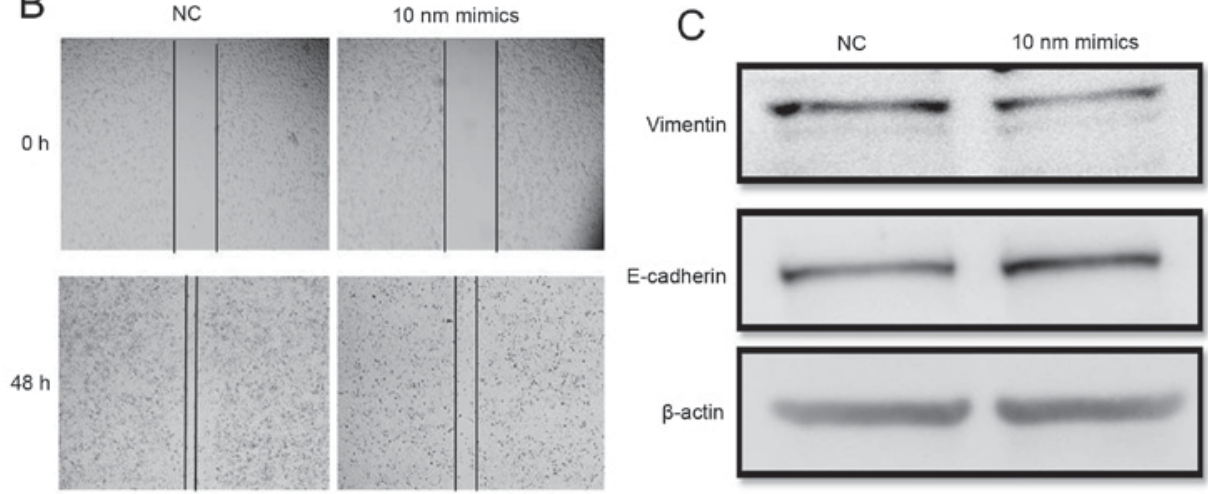

Figure 4. Mmu-miR-294 represses the metastasis of mouse gastric cancer MFC cells by inhibiting vimentin. (A) TargetScan 7.0 online software was used to predict potential factors mediated by mmu-miR-294. Predicted consequential pairing of target region (top) and miRNA (bottom). (B) Wound healing assay for cell migration $48 \mathrm{~h}$ post-transfection with miRNA mimics (magnification, $\mathrm{x} 40$ ). (C) Western blotting for vimentin, E-cadherin and $\beta$-actin proteins $48 \mathrm{~h}$ post-transfection with miRNA mimics. miRNA/miR, microRNA; UTR, untranslated region; NC, negative control.
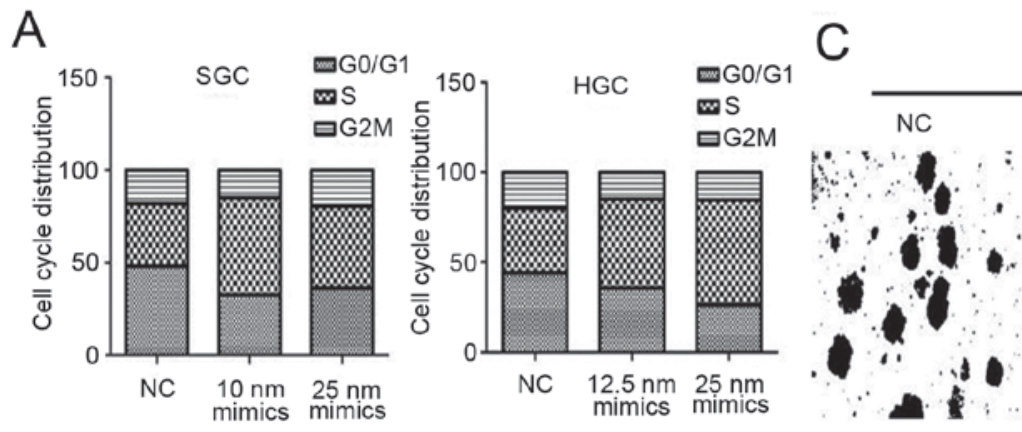

SGC

B
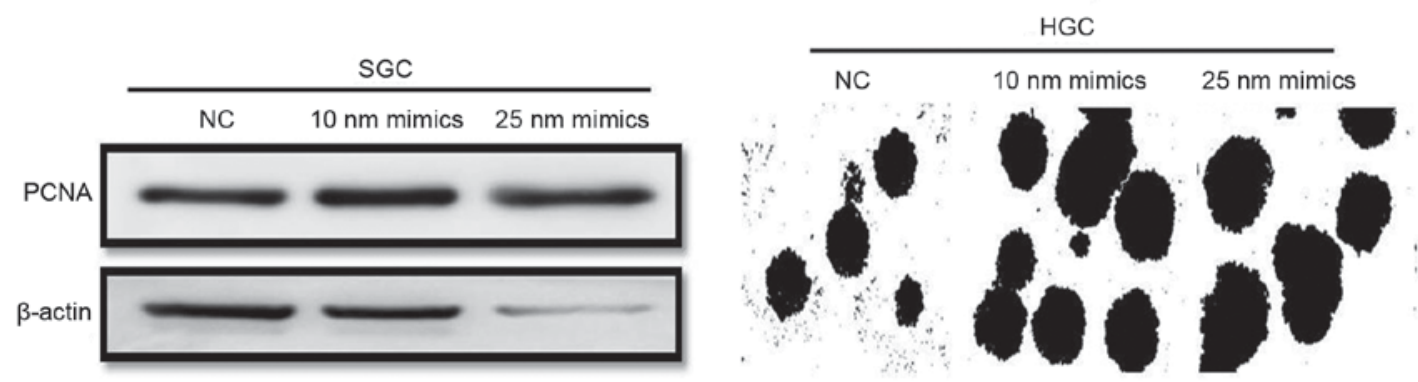

Figure 5. Hsa-miR-373 promotes the proliferation of gastric cancer SGC-7901 and HGC-27 cells. (A) Cell cycle distribution analysis $48 \mathrm{~h}$ post-transfection by flow cytometry. (B) Western blotting for PCNA protein $48 \mathrm{~h}$ post-transfection with miRNA mimics. (C) Cell colony assay for cell proliferation $48 \mathrm{~h}$ post-transfection (magnification, $\mathrm{x} 40$ ). miRNA/miR, microRNA; PCNA, proliferating cell nuclear antigen; NC, negative control.

was used to further investigate the function of miR-373 in mouse gastric cancer metastasis (Fig. 4). Mmu-miR-294 was overexpressed in the mouse gastric cancer MFC cell line. The wound healing results demonstrated that the wound size in miR-294 mimics-treated cells was larger compared with negative control cells at $48 \mathrm{~h}$ post miRNA transfection (Fig. 4B).

To verify the factors mediating the effect of miR-294 in gastric cancer, the target of miR-294 was identified using an online database. The prediction revealed that vimentin mRNA contained a potential miR-294 seed sequence within its 3'-untranslated region (Fig. 4A). Western blotting revealed that the alteration in vimentin expression was inversely associated with miR-294 abundance (Fig. 4C). These results demonstrated that mmu-miR-294 was able to repress the migration of gastric cancer cells by downregulating vimentin expression in vitro.

Hsa-miR-373 promotes the proliferation of gastric cancer SGC-7901 and HGC-27 cells in vitro. miR-373 is able to reduce the metastasis of gastric cancer cells, in addition to promoting tumor cell proliferation under certain conditions. For example, overexpression of miR-373 inhibits proliferation and tumor growth in colon cancer (26) and pancreatic cancer (24), while it promotes proliferation in testicular germ cell cancer (18). It is not clear whether miR-373 is able to promote proliferation 
of gastric cancer cell. The present study investigated the role of miR-373 in gastric cancer cell proliferation. Cell cycle analysis demonstrated that the percentage of cells in the $\mathrm{S}$ phase was increased following treatment with mimics (Fig. 5A). The expression of PCNA in mimics-treated cells, as detected by western blotting, was upregulated markedly (Fig. 5B). The results of the colony-formation assay demonstrated that cells transfected with mimics formed more colonies compared with control cells, and the diameter of colony-formation units in transfected cells was larger compared with control cells (Fig. 5C). The results of the present study demonstrated that miR-373 was able to promote the proliferation of gastric cancer SGC-7901 and HGC-27 cells in vitro.

\section{Discussion}

Gastric cancer is difficult to cure, since patients with gastric cancer frequently exhibit no obvious symptoms in the early stages. The majority of patients with gastric cancer are at the mid-late stages when diagnosed. Over one-half of patients with gastric cancer undergoing radical surgery suffer metastasis, and the long-term survival of such patients is shortened (27). Thus, the exact molecular mechanisms in gastric cancer metastasis require further study.

miRNAs are a type of biomolecule involved in vast range of cellular processes, including proliferation, differentiation, senescence and apoptosis (4-6). The aberrant expression of miRNAs may lead to diseases, including cancer $(28,29)$. miR-373 was first identified to be an oncogene in the tumorigenesis of human testicular germ cell tumors (18), and was upregulated in seminoma compared with normal testicular tissue. The expression of miR-373 was additionally upregulated in breast cancer (30) and hepatocellular carcinoma (31). To elucidate the role of miR-373 in gastric cancer, the expression of miR-373 was analyzed in gastric cancer tissues and their paired non-tumorous tissues by RT-qPCR. The correlation between miR-373 expression and clinical pathological characteristics was subsequently assessed. The data showed that miR-373 is up-regulated in gastric cancer tissue than paired non-tumorous tissues. Furthermore, the miRNA-373 level was negatively correlated with lymph node metastasis.

Accumulating evidence has indicated that miR-373 participates in tumor metastasis (32), and the role of human miR-373 in metastasis is dependent the tissue and organ type. miR-373 was first identified to be a metastasis-promoting miRNA in breast cancer (30). Cluster of differentiation 44 was determined to be a functional target of miR-373 and miR-520c, which was responsible for the migration phenotype (30). In human fibrosarcoma HT1080 cells, miR-373 and miR-520c may promote migration. Serine/threonine-protein kinase mechanistic target of rapamycin and NAD-dependent protein deacetylase sirtuin-1, which are negative regulators of matrix metalloproteinase 9 expression, have been demonstrated to be directly downregulated by miR-373 and miR-520 (33). These previous results illustrated the role of human miR-373 in promoting breast cancer and fibrosarcoma metastasis.

However, miR-373 was additionally demonstrated to function as a suppressor of cell migration and invasion. $\mathrm{Wu}$ et al (34) reported that the overexpression of miR-373 in lung cancer A549 cells decreased migration by targeting
E-cadherin. The hepatitis B antigen, which is involved in HBV-associated hepatocellular carcinoma, was demonstrated to be able to downregulate the expression of miR-373 which consequently reduces E-cadherin expression, suggesting that hepatocellular carcinoma with low miR-373 expression may be more invasive (31). Although these data demonstrated that miR-373 is involved in the metastasis of tumors from different tissues and organs, no clear explanation of the role of miR-373 in gastric cancer metastasis was reported. To examine the impact of miR-373 on the metastasis of gastric cancer, the present study used miRNA mimics to perform gain-of-function experiments. The results demonstrated that miR-373 overexpression was able to inhibit the migration and invasion of gastric cancer SGC-7901 and HGC-27 cells in vitro by inhibiting vimentin expression.

Although it was observed that miR-373 served an important role in the metastasis of gastric cancer, that gastric cancer with a lower level of miR-373 may be more invasive, and that miR-373 overexpression inhibited the migration and invasion of gastric cancer cells by repressing vimentin, the question of whether vimentin is the direct target of miR-373 was not examined in the present study. Therefore, further studies are required to clarify the direct target of miR-373 in gastric cancer.

In conclusion, these findings suggested that miR-373 may be associated with gastric cancer, and that gastric cancer with a lower level of miR-373 may be more invasive. miR-373 may inhibit the metastasis of gastric cancer cells by suppressing the expression level of vimentin. The results of the present study demonstrated an oncogenic role of miR-373 in the metastasis of human gastric cancer, and may provide a novel therapeutic strategy for gastric cancer with lymph node metastasis.

\section{Acknowledgements}

The present study was supported by the National Natural Science Foundation of China (grant nos. 31340040, 81272481, 81270214, 81572075, 81602883 and 81670549), the China Postdoctoral Science Foundation (grant nos. 2015M580403 and 2016T90431), the China Postdoctoral Science Foundation Funded Project (grant no. 2016M600383), the Special Funded Projects of NationalPostdoctoral Fund (grant no.2017T100337), and the Innovation Project for Graduate Student Research of Jiangsu Province (grant no. KYLX15_1096).

\section{References}

1. Brenner H, Rothenbacher D and Arndt V: Epidemiology of stomach cancer. Methods Mol Biol 472: 467-477, 2009.

2. Desai AM, Pareek M, Nightingale PG and Fielding JW: Improving outcomes in gastric cancer over 20 years. Gastric Cancer 7: 196-201, 2004.

3. Crew KD and Neugut AI: Epidemiology of gastric cancer. World J Gastroenterol 12: 354-362, 2006.

4. Krol J, Loedige I and Filipowicz W: The widespread regulation of microRNA biogenesis, function and decay. Nat Rev Genet 11: 597-610, 2010.

5. Mukherji S, Ebert MS, Zheng GX, Tsang JS, Sharp PA and van Oudenaarden A: MicroRNAscan generate thresholds in target gene expression. Nat Genet 43: 854-859, 2011.

6. Pichinuk E, Broday L and Wreschner DH: Endogenous RNA cleavages at the ribosomal SRL site likely reflect miRNA mediated translational suppression. Biochem Biophys Res Commun 414: 706-711, 2011. 
7. Calin GA and Croce CM: MicroRNA signatures in human cancers. Nat Rev Cancer 6: 857-866, 2006.

8. Wang $\mathrm{M}, \mathrm{Gu} \mathrm{H}$, Qian $\mathrm{H}$, Zhu W, Zhao C, Zhang $\mathrm{X}$, Tao Y, Zhang $\mathrm{L}$ and $\mathrm{Xu} \mathrm{W}$ : $\mathrm{miR}-17-5 \mathrm{p} / 20 \mathrm{a}$ are important markers for gastric cancer and murine double minute 2 participates in their functional regulation. Eur J Cancer 49: 2010-2021, 2013.

9. Mitash N, Agnihotri S, Tiwari S, Agrawal V and Mandhani A: MicroRNA-21 could be a molecular marker to predict the recurrence of nonmuscle invasive bladder cancer. Indian J Urol 33 283-290, 2017

10. Jiao W, Leng X, Zhou Q, Wu Y, Sun L, Tan Y, Ni H, Dong X, Shen T, Liu Y and Li J: Different miR-21-3p isoforms and their different features in colorectal cancer. Int J Cancer 141: 2103-2111, 2017.

11. Song Y, Zuo Y, Qian XL, Chen ZP, Wang SK, Song L and Peng LP: Inhibition of MicroRNA-21-5p promotes the radiation sensitivity of non-small cell lung cancer through HMSH2. Cell Physiol Biochem 43: 1258-1272, 2017.

12. Motoyama K, Inoue H, Mimori K, Tanaka F, Kojima K, Uetake H, Sugihara K and Mori M: Clinicopathological and prognostic significance of PDCD4 and microRNA-21 in human gastric cancer. Int J Oncol 36: 1089-1095, 2010.

13. Wu Y, Song Y, Xiong Y, Wang X, Xu K, Han B, Bai Y, Li L, Zhang Y and Zhou L: MicroRNA-21 (Mir-21) promotes cell growth and invasion by repressing tumor suppressor PTEN in colorectal cancer. Cell Physiol Biochem 43 945-958, 2017.

14. Zhang BG, Li JF, Yu BQ, Zhu ZG, Liu BY and Yan M: microRNA-21 promotes tumor proliferation and invasion in gastric cancer by targeting PTEN. Oncol Rep 27: 1019-1026, 2012.

15. Wang L, Qu J, Zhou L, Liao F and Wang J: MicroRNA-373 inhibits cell proliferation and invasion via targeting BRF2 in human non-small cell lung cancer A549 cell line. Cancer Res Treat: Oct 12, 2017 (Epub ahead of print).

16. Li Y, Zhang D and Wang J: MicroRNA-373 promotes tumorigenesis of renal cell carcinoma in vitro and in vivo. Mol Med Rep Sep 16: 7048-7055, 2017.

17. Hua Y, Chen H, Wang L, Wang F, Wang P, Ning Z, Li Y, Liu L, Chen $\mathrm{Z}$ and Meng Z: Low serum miR-373 predicts poor prognosis in patients with pancreatic cancer. Cancer Biomark 20 95-100, 2017.

18. Suh MR, Lee Y, Kim JY, Kim SK, Moon SH, Lee JY, Cha KY, Chung HM, Yoon HS, Moon SY, et al: Human embryonic stem cells express a unique set of microRNAs. Dev Biol 270: 488-498, 2004.

19. Zhang X, Yan Z, Zhang J, Gong L, Li W, Cui J, Liu Y, Gao Z, Li J, Shen L and Lu Y: Combination of hsa-miR-375 and hsa-miR-142-5p as a predictor for recurrence risk in gastric cancer patients following surgical resection. Ann Oncol 22: 2257-2266, 2011
20. Yan Z, Xiong Y, Xu W, Li M, Cheng Y, Chen F, Ding S, Xu H and Zheng G: Identification of recurrence-related genes by integrating microRNA and gene expression profiling of gastriccancer. Int J Oncol 41: 2166-2174, 2012.

21. Zhang X, Li X, Tan Z, Liu X, Yang C, Ding X, Hu X, Zhou J, Xiang S, Zhou C and Zhang J: MicroRNA-373 is upregulated and targets TNFAIP1 in human gastric cancer, contributing to tumorigenesis. Oncol Lett 6: 1427-1434, 2013.

22. Kim KS, Kim JS, Lee MR, Jeong HS and Kim J: A study of microRNAs in silico and in vivo: Emerging regulators of embryonic stem cells. FEBS J 276: 2140-2149, 2009.

23. Livak KJ and Schmittgen TD: Analysis of relative gene expression data using real-time quantitative PCR and the 2(-Delta Delta C(T)) method. Methods 25: 402-408, 2001

24. Nakata K, Ohuchida K, Mizumoto K, Aishima S, Oda Y, Nagai E and Tanaka M: MicroRNA-373 is down-regulated in pancreatic cancer and inhibits cancer cell invasion. Ann Surg Oncol 21 (Suppl 4): S564-S574, 2014

25. Peng Z, Wang CX, Fang EH, Wang GB and Tong Q: Role of epithelial-mesenchymal transition in gastric cancer initiation and progression. World J Gastroenterol 20: 5403-5410, 2014.

26. Tanaka T, Arai M, Wu S, Kanda T, Miyauchi H, Imazeki F, Matsubara $\mathrm{H}$ and Yokosuka O: Epigenetic silencing of microRNA-373 plays an important role in regulating cell proliferation in colon cancer. Oncol Rep 26: 1329-1335, 2011.

27. Cao Y, DePinho RA, Ernst M and Vousden K: Cancer research: Past, present and future. Nat Rev Cancer 11: 749-754, 2011.

28. Han C, Shen JK, Hornicek FJ, Kan Q and Duan Z: Regulation of microRNA-1 (miR-1)expression in human cancer. Biochim Biophys Acta 1860: 227-232, 2017.

29. Matsuda A, Yan IK, Foye C, Parasramka M and Patel T: MicroRNAs as paracrine signaling mediators in cancers and metablic diseases. Best Pract Res Clin Endocrinol Metab 30: 577-590, 2016

30. Huang Q, Gumireddy K, Schrier M, le Sage C, Nagel R, Nair S, Egan DA, Li A, Huang G, Klein-Szanto AJ, et al: The microRNAs miR-373 and miR-520 c promote tumour invasion and metastasis. Nat Cell Bio 10: 202-210, 2008.

31. Arzumanyan A, Friedman T, Kotei E, Ng IO, Lian Z and Feitelson MA: Epigeneticrepression of E-cadherin expression by hepatitis B virus x antigen in liver cancer. Oncogene 31: 563-572, 2012.

32. Stacy AJ, Craig MP, Sakaram S and Kadakia M: $\Delta N p 63 \alpha$ and microRNAs: Leveraging the epithelial-mesenchymal transition. Oncotarget 8: 2114-2129, 2017

33. Liu P and Wilson MJ: miR-520c and miR-373 upregulate MMP9 expression by targeting mTOR and SIRT1 and activate the Ras/Raf/MEK/Erk signaling pathway and NF- $\kappa \mathrm{B}$ factor in human fibrosarcoma cells. J Cell Physiol 227: 867-876, 2012.

34. Wu W, He X, Kong J and Ye B: Mir-373 affects human lung cancer cells' growth and its E-cadherin expression. Oncol Res 20: 163-170, 2012. 